\title{
Epidemiology and in vitro susceptibility of Fusarium species from human infections in Germany - results of a retrospective multicenter study
}

Axel G. Hamprecht ${ }^{1}$, Stephan Göttig ${ }^{2}$, Andrea Haas ${ }^{3}$, Jürgen Held ${ }^{4}$, Martin Koeppel ${ }^{3}$, Oliver Kurzai ${ }^{5}$, Ahmad Saleh ${ }^{1}$, Michael Seibold ${ }^{6}$, Jörg Steinmann, Katrin Tintelnot ${ }^{6}$, Grit Walther ${ }^{5}$, Michael Weig ${ }^{8}$ and Volker Rickerts ${ }^{6}$

${ }^{1}$ Institute for Medical Microbiology, Immunology and Hygiene, University Hospital Cologne, Germany; ${ }^{2}$ Institute for Medical Microbiology and Infection Control; University Hospital Frankfurt; Frankfurt am Main, Germany; ${ }^{3}$ Max-von-Pettenkofer Institute, LMU Munich, Munich, Germany; ${ }^{4}$ Klinische Mikrobiologie, Immunologie und Hygiene, Universitätsklinikum Erlangen, Erlangen, Germany; ${ }^{5}$ Nationales Referenzzentrum für Invasive Pilzinfektionen NRZMyk, Jena, Germany; ${ }^{6}$ Robert Koch Institute, FG 16 , Berlin, Germany; ${ }^{7}$ Institute of Medical Microbiology, University Hospital Essen, University of Duisburg-Essen, Essen, Germany; ${ }^{8}$ nstitute for Medical Microbiology, University Medical Center Göttingen, Göttingen, Germany

\section{Background}

- Fusarium spp. cause severe infections in immunocompromised and immune-competent hosts

- disseminated infections in hemato-oncology patients associated with a mortality of $90-100 \%$

- high resistance rate

- epidemiology of fusariosis in Germany unknown

Objectives

- determine the epidemiology and susceptibility of Fusarium spp. from human infections in Germany

Methods

- 85 clinical strains isolated 1999-2017 at seven medical centers in Germany were included in the study

- identification was carried out by using morphology, mass spectrometry and molecular methods (TEF1a, ITS and RPB2 sequencing)

- Susceptibility testing by EUCAST microdilution reference method

\section{Results}

- 28 isolates (32.9\%) were from blood stream infections (BSI), 20 (23.5\%) from respiratory infections, 19 (22.4\%) from skin and soft tissue infections (SSTI), 15 (17.6\%) from eye and 3 (3.5\%) from other infections

- F. fujikuroi sp. comp. (FFSC) most frequent species complex $(47.1 \%)$, followed by $F$. solani sp. complex (FSSC), Table 1

- FFSC most frequent SC in BSI and respiratory infections, whereas FSSC was more common in SSTI and eye infections, Figure 1

- Overall $F$. proliferatum most common species in invasive infections and $F$. musae the most frequent species in BSI

- Susceptibility varies greatly among clinical isolates, even within the same species/species complex (SC)

- FFSC isolates showed higher amphotericin B but lower voriconazole MICs compared to FSSC, Table 2

Conclusion

- FFSC and FSSC are the most frequently identified SC from human infections in Germany

- F. musae seems to be an emerging species in BSI and reported for the first time in Germany

- species identification not sufficient for the prediction of susceptibility; susceptibility testing is required to optimize therapy, ideally using a microdilution method

\begin{tabular}{|lcc|} 
& $\mathrm{n}$ & $\%$ \\
\hline F. fujikuroi sp. comp. (FFSC) & $\mathbf{4 0}$ & $\mathbf{4 7 . 1}$ \\
F. musae & 12 & 14.1 \\
F. verticillioides & 1 & 1.2 \\
F. proliferatum & 26 & 30.6 \\
F. sacchari & 1 & 1.2 \\
\hline F. oxysporum sp. comp. (FOSC) & $\mathbf{1 2}$ & $\mathbf{1 4 . 1}$ \\
\hline F. dimerum sp. comp. (FDSC) & $\mathbf{3}$ & $\mathbf{3 . 5}$ \\
F. delphinoides & 1 & 1.2 \\
F. dimerum & 2 & 2.4 \\
\hline F. solani sp. comp. (FSSC) & $\mathbf{3 0}$ & $\mathbf{3 5 . 3}$ \\
F. keratoplasticum & 3 & 3.5 \\
\hline F. petroliphilum & 16 & 18.8 \\
other FSSC & 11 & 12,9 \\
\hline Total & $\mathbf{8 5}$ & $\mathbf{1 0 0}$ \\
\hline
\end{tabular}

Table 1 Species distribution of Fusarium spp. from human infections

\section{Origin of isolates}

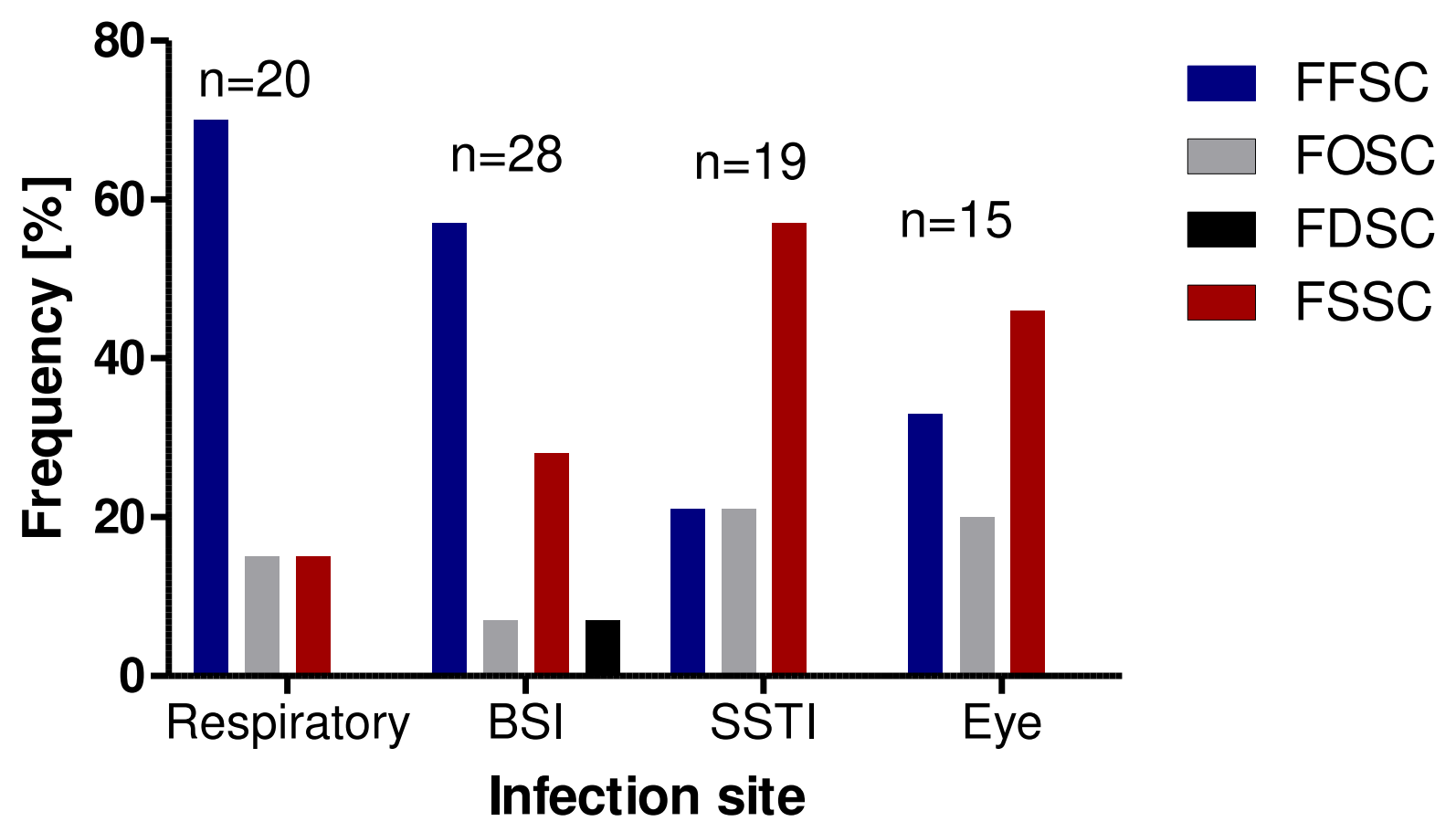

Figure 1 Representation of species complexes depending on infection site; BSI: blood stream infections; SSTI: skin and soft tissue infection

\begin{tabular}{llcccc} 
FFSC & Range & $0.06->64$ & $2->16$ & $0.5->16$ & $0.5->8$ \\
$(\mathrm{n}=38)$ & Median & 4 & 16 & 2 & 8 \\
FOSC & Range & $1-16$ & $4->16$ & $2->16$ & $2->8$ \\
$(\mathrm{n}=12)$ & Median & 1 & $>16$ & 2 & 8 \\
FDSC & Range & $0.5-16$ & $>16$ & 0.5 & $2-8$ \\
$(\mathrm{n}=2)$ & Median & 8.25 & $>16$ & 0.5 & 5 \\
FSSC & Range & $0.125-2$ & $>16$ & $1->16$ & $1->8$ \\
$(\mathrm{n}=30)$ & Median & 1 & $>16$ & 8 & $>16$ \\
\hline
\end{tabular}

Table 2 Antifungal susceptibility of tested Fusarium isolates APB: amphotericin B; ITC: itraconazole; VRC: voriconazole; POS: posaconazole

\section{Contact information}

Axel Hamprecht, Institute for Medical Microbiology, Immunology and Hygiene, D-50935 Cologne/Germany; e-mail: axel.hamprecht@uk-koeln.de 